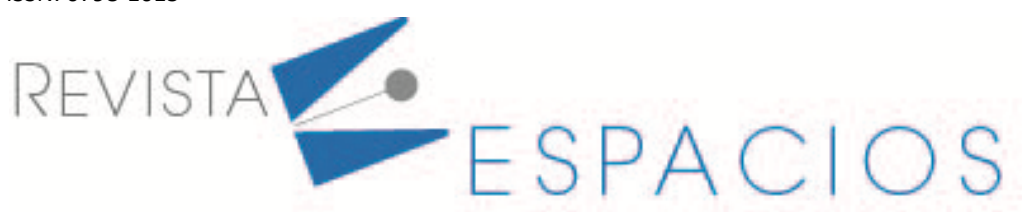

\title{
Sistema de gestión de abastecimiento para la asociación de artesanos del Sugamuxi (Colombia)
}

\section{Supply management system for the Sugamuxi artisans association}

\author{
MESA, Josué I. ${ }^{1}$ \\ SALAZAR, Hugo F. ${ }^{2}$

\section{Resumen} \\ Esta investigación tiene como objetivo definir las políticas de gestión de inventarios para el sistema de \\ gestión abastecimiento de la asociación de productores artesanales de la provincia del Sugamuxi, bajo \\ el concepto de Vendor Managed Inventory. Fue necesario identificar las materias primas utilizadas por \\ los artesanos y su comportamiento de consumo, para luego estimar los costos asociados a inventarios, \\ seleccionar proveedores y generar políticas de gestión de inventarios utilizando modelos \\ determinísticos y estocásticos, seleccionando la política que genera menor costo. \\ Palabras clave: abastecimiento, gestión inventarios, modelos determinísticos, modelos estocásticos, \\ VMI.
}

\begin{abstract}
This research aims to define the inventory management policies for the supply management system of the association of artisan producers of the province of Sugamuxi, under the concept of Vendor Managed Inventory. It was necessary to identify the raw materials used by the artisans and their consumption behavior, in order to estimate the costs associated with inventories, select suppliers and generate inventory management policies using deterministic and stochastic models, selecting the policy that generates the lowest cost.

Key words: supplying, inventory management, deterministic models, stochastic models, VMI.
\end{abstract}

\section{Introducción}

La asociación de artesanos productores de la provincia del Sugamuxi (Boyacá, Colombia) (APROARTES) compuesta por 61 artesanos tiene como propósito promover estrategias colaborativas de abastecimiento, producción y distribución a las unidades productivas que la conforman. Se ha identificado el abastecimiento colaborativo como uno de las alternativas para solucionar los problemas en el uso de los recursos de los miembros de la asociación, determinando que un sistema de gestión de abastecimiento de materias primas e insumos colaborativo es de vital importancia en APROARTES.

Los requerimientos de mejoramiento de APROARTES, siguen el comportamiento de la economía a nivel nacional e internacional, que ha hecho que las empresas u organizaciones tengan que ser día a día más competitivas si

\footnotetext{
${ }^{1}$ Docente Escuela de Administración Industrial en la Universidad Pedagógica y Tecnológica de Colombia. Estudiante Maestría en Ingeniería con énfasis en Ingeniería Industrial, Universidad Pedagógica y Tecnológica de Colombia. Especialista en Ingeniería de Producción y Operaciones, Universidad Pedagógica y Tecnológica de Colombia. Ingeniero Industrial, Universidad Pedagógica y Tecnológica de Colombia. josue.mesa@uptc.edu.co

${ }^{2}$ Docente Escuela de Ingeniería Industrial en la Universidad Pedagógica y Tecnológica de Colombia. Magister en Ingeniería Industrial, Universidad de Distrital Francisco José de Caldas. Ingeniero Industrial, Universidad Industrial de Santander. hugo.salazar@uptc.edu.co
} 
quieren sobrevivir en el tiempo, por lo tanto, las empresas u organizaciones han tenido que mejorar y volver más eficiente su gestión de abastecimiento, logrando disminuir los costos y aumentar el nivel de servicio ofrecido al cliente (Díaz-Batista \& Pérez-Armayo, 2012). Complementariamente, Arango-Serna, Adarme-Jaimes y ZapataCortes (2010) mencionan que los sistemas de gestión de abastecimiento permiten mejorar la eficiencia en la adquisición de materias primas o insumos, y a su vez, disminuir los costos asociados al abastecimiento y el manejo de inventarios.

El sistema de gestión de abastecimiento comprende tres subfunciones, las cuales son: gestión de compras, recepción y almacenamiento (Pereira, Oliveira, \& Carravilla, 2020), donde cada una de estas subfunciones tiene retos y decisiones a tomar. En la gestión de compras, se tiene que tomar la decisión de seleccionar proveedores, la cual es de vital importancia, dado que se tiene como reto la adquisición de materias primas o insumos de calidad y en las cantidades necesarias para los procesos productivos (Gómez, Cano, \& Campo, 2016); además, seleccionar proveedores de manera correcta permite dar una respuesta oportuna a los requerimientos de los clientes, y a su vez, mejorar la competitividad de las organizaciones (Alfonso, 2018).

La eficiencia en la gestión de inventarios según Barcelli-Gómez (2017) es de vital importancia dado que permite aumentar el nivel de servicio ofrecido al cliente, generar una disminución de costos operativos y aumentar la competitividad de las empresas. No obstante lograr equilibrio entre el nivel de servicio y los costos por la gestión de inventarios es el gran desafío de las empresas, como lo menciona Duran (2012) ofrecer un nivel de servicio mayor afecta los costos por manejos de inventarios de forma directa. Sin embargo, como se evidencia en la investigación de Peña y Silva (2015) tener grandes cantidades de inventarios afectan los costos de los productos, lo cual disminuye la competitividad de las empresas y genera la pérdida de clientes, y a su vez, el continuo cambio del mercado y el ciclo de vida de los productos hacen que las empresas evalúen continuamente si sus procesos de gestión de inventarios son eficientes (Aguilar Santamaría, 2012). Adicionalmente, Osorio (2015) menciona que la gestión de inventarios puede llegar a ser muy compleja por la incertidumbre que la rodea y los productos que se gestionan, llevando a las empresas a tener exceso de productos en inventarios o faltantes de productos.

La investigación sobre el sistema de abastecimiento se evidenció en el trabajo de campo del proyecto institucional de la Universidad Pedagógica y Tecnológica de Colombia (UPTC), "Direccionamiento estratégico para la asociación de productores artesanales de la provincia del Sugamuxi-APROARTES", donde se identificó que APROARTES no tiene un sistema de gestión de abastecimiento para adquirir sus materias primas e insumos, puesto que el abastecimiento se realiza con base en la estimación empírica de cada artesano, sin tener en cuenta la incertidumbre de la demanda. Además, se identificó que los artesanos realizan de dos formas diferentes la compra de sus materias primas, por compra en punto de comercio $(84 \%)$ y por pedido a proveedor $(16 \%)$, definiendo las cantidades de pedido en una forma aproximada según su experiencia.

Adicionalmente, los artesanos no asocian todos los costos en que incurren al realizar el abastecimiento de sus materias primas e insumos, lo cual genera que no tengan control en los costos generados por la gestión de sus inventarios y al no tener una correcta estimación no permite evaluar la gestión de abastecimiento de APROARTES (Taboada-González, Aguilar-Virgen, Ibarra-TrujiMo \& Ramírez-Barreto, 2016). Complementariamente, APROARTES no cuenta con una estimación de la demanda de sus productos que se ajuste al comportamiento real de sus ventas, por lo tanto, toda la gestión de abastecimiento se ve afectada por los faltantes o excesos en las materias primas e insumos utilizados en los productos que comercian y como consecuencia, se han generado faltantes o exceso de los mismos, incurriendo en una disminución del nivel de servicio ofrecido a los clientes. Una consecuencia directa de no tener una adecuada gestión de abastecimiento es la pérdida de clientes debido a los altos costos de los productos, y esto queda evidenciado en la disminución de sus ventas, volviendo a los artesanos no competitivos frente a los nuevos mercados emergentes que incurren en la región (Peña \& Silva, 2015). 
Por lo anterior, esta investigación tiene como objetivo analizar políticas de gestión de inventarios para el sistema de gestión abastecimiento colaborativo de las materias primas de APROARTES, de tal manera que permita disminuir los costos por abastecimiento y aumentar el nivel de servicio ofrecido al cliente.

Para desarrollar el objetivo anterior, se identificaron aspectos fundamentales que influyen en los sistemas de gestión de abastecimiento y en las políticas de gestión de inventarios colaborativos, por lo tanto, se identificaron características de la metodología Vendor Managed Inventory (VMI), las cuales se utilizan como base para el diseño de las políticas de gestión de inventarios, donde algunos aspectos fundamentales son: productos, materias primas, insumos, consumos y proveedores. A su vez, la identificación de estos aspectos fundamentales permitió seleccionar las materias primas o insumos que harán parte del sistema de gestión de abastecimiento colaborativo, para las cuales se determinó el comportamiento del consumo mediante el uso de estadística descriptiva e inferencial.

Se seleccionaron proveedores utilizando el método Pugh, incluyendo criterios como el precio, la calidad, tiempos de entrega, entre otros; se definieron las cantidades a pedir a los proveedores utilizando modelos determinísticos y estocásticos, los cuales serán la base para la formulación de políticas de manejo de inventarios y se seleccionó la mejor política de manejo de inventarios para APROARTES, teniendo como criterio de selección el menor costo anual del sistema de gestión de inventarios.

\section{Metodología}

Para el sistema de abastecimiento de APROARTES se utilizó una metodología compuesta por 4 fases:

1. Estado del arte: sé realiza una revisión relacionada con los sistemas de gestión de abastecimiento y las políticas de gestión de inventarios.

2. Investigación para proponer el sistema de abastecimiento colaborativo de APROARTES utilizando VMI, fue necesario recolectar en campo información del funcionamiento de APROARTES y los artesanos que la componen, como parte del proyecto macro "Direccionamiento estratégico para la asociación de productores artesanales de la provincia del Sugamuxi-APROARTES".

3. Análisis de toda la información recolectada en la investigación, se seleccionan las materias primas de abastecimiento y se define su comportamiento de consumo.

4. Generación de políticas: se identifican los proveedores de las materias primas seleccionados, se realiza el cálculo de los costos que influyen en los modelos de generación de políticas de abastecimiento, y se formularán políticas de gestión de inventarios a partir de modelos determinísticos y estocásticos.

\section{Estado del arte}

Como punto de partida se decide realizar una búsqueda de investigaciones relacionas con las tres subfunciones de los sistemas de gestión de abastecimiento: gestión de compras, recepción y almacenamiento (incluye el manejo de inventarios) (Pereira, Oliveira, \& Carravilla, 2020). Se identificaron metodologías, herramientas, variables y parámetros que influyen en las sub funciones de los sistemas de gestión de abastecimiento y se realizó en bases de datos como SCOPUS, ProQuest, Science Direct, SciELO y Google académico.

Una metodología para la evaluación y selección de proveedores utilizando lógica difusa en el sector minero del oro, donde se consideran características técnicas de los insumos, calidad, capacidad de producción, tiempos de entrega, precios, entre otras, se describe en la investigación de Gómez et al. (2016). Sin embargo, Alfonso (2018) utiliza en su investigación para la selección de proveedores un proceso de análisis jerárquico y un modelo de programación lineal entera mixta, y a su vez, propone una metodología basada en optimización para la selección 
de proveedores, y se complementa con el método de selección y evaluación de proveedores el método Pugh, el cual ha sido utilizado en el sector de servicios de mantenimiento (García Gómez, 2013).

Torrijos (2018) realiza una valoración de los proveedores basada en el análisis dimensional, permitiendo tener en cuenta múltiples criterios para la selección de los proveedores, además en su investigación utiliza una metodología multicriterio para realizar la selección de proveedores, donde se incluyen criterios como calidad, precios y tiempos de entrega. Adicionalmente, Amaro Castillo (2017) utiliza una metodología para la selección de proveedores en la que formula criterios de decisión específicos para proveedores del sector transporte. De igual manera, se han utilizado las herramientas Analytic Hierarchy Process (AHP) y Analytic Network Process (ANP) para tomar decisiones de selección de proveedores con múltiples criterios (Muñoz Claro, 2014).

Para las características de recepción y almacenamiento, donde se incluye el manejo de inventarios, Parga-Prieto y Aranda-Pinilla (2018) identifican la política de inventarios como uno de los factores claves para el éxito, aplicando como método para definir los periodos de abastecimiento las heurísticas: Silver-Meal, costo unitario mínimo y Wagner-Whitin. Por otro lado, un claro ejemplo de la importancia de la política de inventarios son los resultados obtenidos por Escobar, Linfati y Jaimes (2017) quienes plantearon una política de inventarios que toma como metodología la simulación de Montecarlo implementada en Microsoft Excel, con la que se logró aumentar la eficiencia del manejo de inventarios y de esta manera incrementar el nivel de servicio ofrecido al cliente y la disminución de los costos de operación.

En la investigación de Castellano De La Cruz, Gamarra Vengoechea y Garcia Luenga (2017) identifican el aprovisionamiento conjunto como una de las políticas que aumenta la eficiencia de la gestión de inventarios, y utilizan la simulación mediante el software Arena para verificar la eficiencia de esta política, y se identificó que la gestión de inventarios colaborativa logra generar valor compartido entre los agentes de la cadena.

Adicionalmente, Palacio-León, Barrios-León y Adarme-Jaimes (2018) incluyen el concepto de productividad saludable a la gestión de inventarios colaborativos, la cual permite ofrecer niveles de servicio por encima del 85\%. Según Sablón-Cossío, Julia Acevedo-Urquiaga, Acevedo-Suárez y Medina-León (2015) una de las posibles barreras para implementar una gestión de inventarios conjunta es el intercambio de información inter empresarial, y mencionan: la planificación, los pronósticos y el reaprovisionamiento colaborativo como método para la generación de la política de inventario.

En referencia a lo anterior Salas-Navarro, Miguel-Mejía y Acevedo-Chedid (2017) mencionan que la gestión de inventarios colaborativos se debe desarrollar mediante la metodología de gestión de inventarios que se aprecia en la Figura 1, logrando un alto aumento en las utilidades teniendo en cuenta: políticas de contratación, excelencia de procesos y el servicio al cliente.

De manera complementaria en la investigación de Lancheros, Mojica y Barbosa (2015) se evidencia el uso de técnicas de minería de datos para analizar patrones ocultos de comportamiento en el departamento de inventarios, como método funcional para el descubrimiento de conocimiento en el área, y poder determinar políticas de inventarios que aumenten el nivel de servicio al cliente y disminuyan los costos de operativos.

Silador Utrera, Naranjo García, Marrero Marrero, Utrera Velázquez y Rodríguez Pacha (2015) utilizan el modelo clásico de cantidad económica de pedido (EOQ), aumentando el nivel del servicio y disminuyendo los costos asociados a la gestión de inventarios. Adicionalmente, Rodríguez (2015) describe el modelo EOQ como uno de los modelos más sencillos y fundamentales para la gestión de inventarios. 
Figura 1

Metodología gestión de inventarios colaborativos

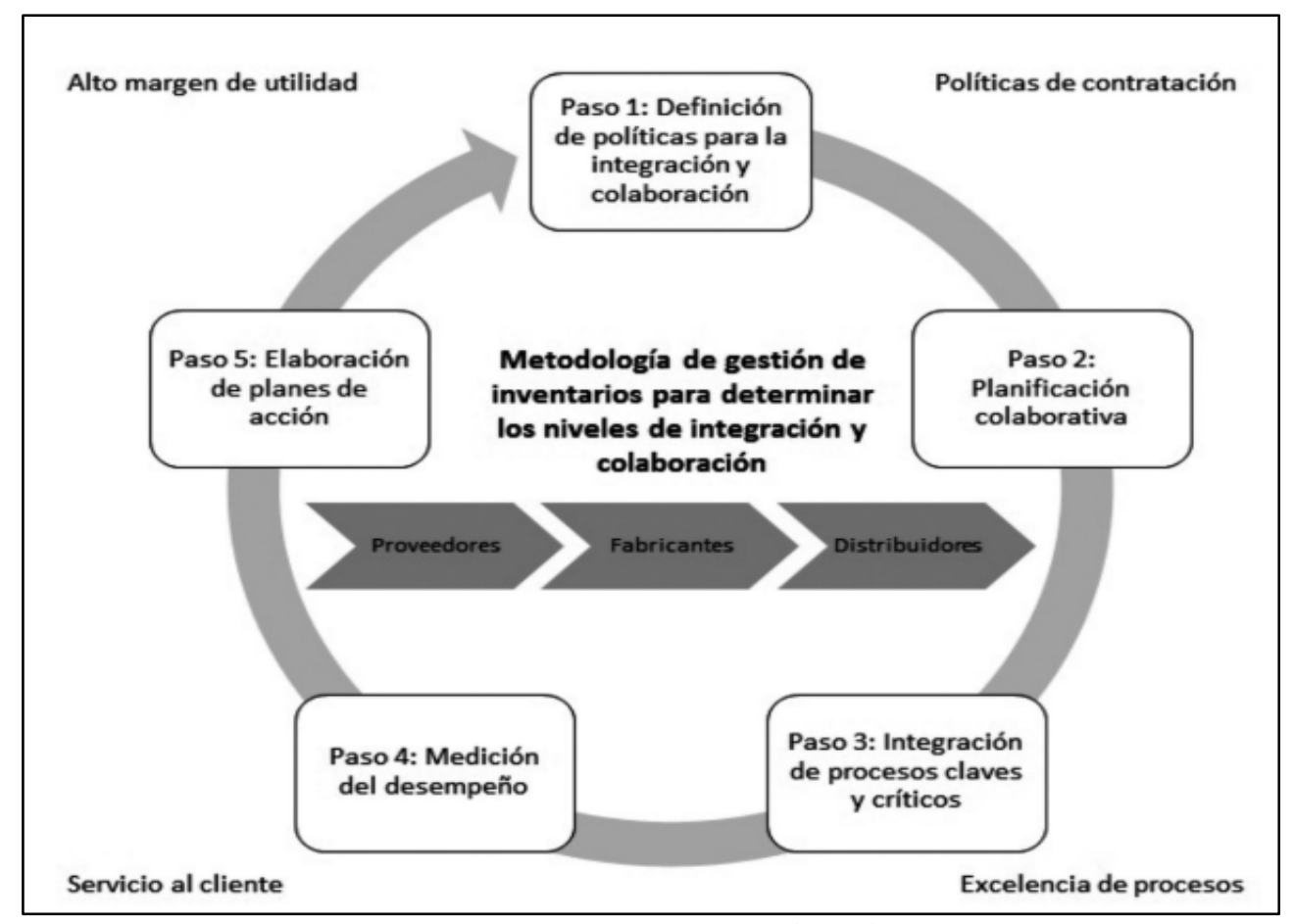

Fuente: Salas-Navarro et al. (2017)

Razmi, Hosseini Rad y Sangari (2010) plantean varios escenarios de integración entre cliente y proveedor y utilizan modelos matemáticos para determinar las cantidades a enviar al cliente y los niveles de inventario a manejar, y Southard y Swenseth (2008) desarrollan un modelo de simulación de eventos discretos en Promodel que plantea diversos escenarios para la entrega de combustible en el sector agrícola, donde se tiene varios clientes que son las cooperativas, por lo tanto plantea VMI para la determinación de los niveles de inventario.

De igual manera Tat, Taleizadeh y Esmaeili (2015) desarrollan un modelo EOQ para artículos deteriorados no instantáneos administrados por el proveedor y evalúa dos escenarios: se permite escasez y no se permite escasez. Complementariamente, Zanoni y Jaber (2015) consideran un VMI con envío, en el cual el proveedor mantiene la propiedad del inventario hasta que el comprador lo retira de inventario, y se ofrece al proveedor la posibilidad de revisar los planes de producción y envío para planificar las diferentes políticas de inventarios.

Sin embargo, para tener una política de inventario eficiente como mencionan Pérez-Vergara, Cifuentes-Laguna, Vásquez-García y Marcela-Ocampo (2013) es necesario identificar las causas raíz que generan la baja eficiencia de la gestión de inventarios, asociando como causa raíz la no correcta identificación de los costos en la gestión de inventarios, quedando evidenciado en la investigación de Taboada-González et al. (2016) donde se identifican que la no asociación de todos los costos en que se incurren al realizar la gestión de inventarios genera no tener control en los costos de la gestión de sus inventarios en los concesionarios de demanda baja y media.

Complementariamente en Peña y Silva (2015) identifican la no correcta asociación de costos a la gestión de inventarios como causa raíz para no lograr una gestión de inventarios eficiente y, por lo tanto, como logra afectar directamente el nivel del servicio ofrecido al cliente y disminuir la competitividad de las empresas. Sin embargo, Sánchez y Ramírez (2018) identifican que en algunos sistemas existe la generación de costos adicionales dependiendo del producto que se almacena y, por lo tanto, deben de tenerse en cuenta los costos por deterioro de los productos para poder tener políticas de gestión de inventarios eficientes. 
También, Bustamante-Salazar (2017) plantea el modelo de costeo ABC como modelo determinístico para la gestión de inventarios, el cual busca asignar de manera más precisa los costos y el control de los recursos. Adicionalmente, Vázquez Yánez (2012) analiza la implementación del costeo ABC en la empresa Martec Cía. Ltda y como este sistema de costeo aumentó las utilidades de la empresa ya mencionada. Taboada-González et al. (2016) utilizaron el modelo ABC para lograr la disminución de costos en concesionarios de baja y media demanda, mencionando que no se requieren aplicar técnicas de análisis muy sofisticadas para lograr la disminución de costos en la gestión de inventarios.

De otro lado, Castellano De La Cruz et al. (2017) identifican que una de las causas raíz que generan la baja eficiencia del manejo de inventarios es la no correcta estimación de la demanda, la cual se puede ver afectada por el error de pronóstico, tal como lo indica Arias-Vargas (2017), cuya investigación demostró que al utilizar un modelo de estimación que genere un error de pronóstico bajo, se puede lograr una disminución en el inventario de seguridad hasta de un $40 \%$ y la disminución de productos faltantes o en exceso. También Gutiérrez y Rodríguez (2008) identifican la no correcta estimación de la demanda en empresas del departamento de Antioquia, y como consecuencia se generó un desnivel en los inventarios afectando el nivel de servicio al no tener los productos suficientes para suplir la demanda.

De lo anterior se puede concluir que los sistemas de gestión de abastecimiento están compuestos por tres subfunciones, las cuales son: gestión de compras, recepción y almacenamiento, las cuales se deberán contemplar y analizar en el desarrollo de la investigación. A su vez, se identifica el método Pugh como método eficiente para la selección de proveedores, debido a que permite cuantificar los criterios de selección de los proveedores. Por último, el estado del arte permitió identificar modelos determinísticos (EOQ y programación lineal) y estocásticos (EOQ, pedidos conjuntos y lógica difusa) para realizar la gestión de inventarios aplicable al VMI, donde la selección del tipo de modelo dependerá del comportamiento de la demanda.

\section{Generación de políticas}

En esta sección del artículo se desarrollará la fase de análisis y la fase de generación de políticas, las cuales son parte de la metodología. Por lo tanto, se seleccionarán las materias primas o insumos a hacer parte del sistema de abastecimiento y se estimará el comportamiento de estas; se formularán modelos para generar políticas de gestión de inventarios para cada una de las materias primas seleccionadas, por lo que, se identifican costos, proveedores y modelos a utilizar.

\subsection{Selección de materias primas o insumos}

Dado que APROARTES está compuesta por 61 artesanos de la provincia del Sugamuxi, los cuales producen 175 productos representativos y para los cuales utilizan aproximadamente 92 materias primas o insumos, fue necesario identificar las materias primas o insumos que más se utilizan y consumen en APROARTES. En la Figura 2 se relaciona la cantidad de productos que se fabrican a partir de las 10 materias primas más comunes, para luego seleccionar las materias primas que formaran parte del sistema de gestión de abastecimiento, teniendo como criterios de selección el mayor consumo de las materias primas y características similares de estas (forma, uso, almacenamiento, peso y volumen. Como resultado de aplicación del criterio anterior se seleccionan la lana natural, la lana sintética y la media lana para formar parte del sistema de gestión de abastecimiento. 
Figura 2

Cantidad de productos por materia prima

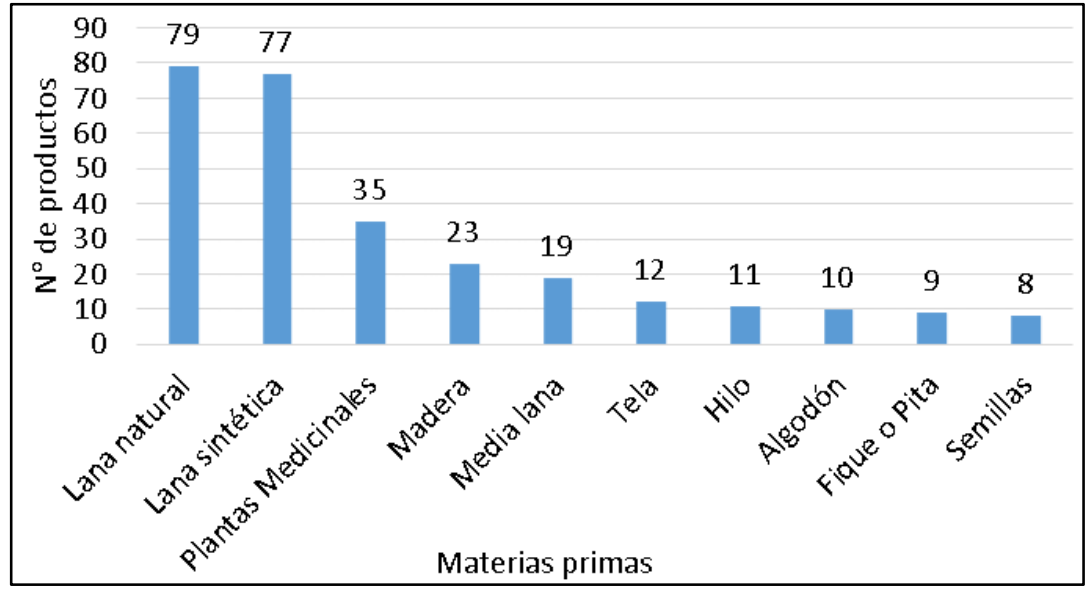

Fuente: elaboración propia

\subsection{Comportamiento consumo de materias primas}

Después de seleccionar la lana natural, la lana sintética y la media lana como las materias primas que formaran parte del sistema de abastecimiento, se procedió a definir el comportamiento, analizando toda la información de campo recolectada de los consumos, identificando que 19 artesanos de los 61 utilizan estás materias primas y se recolectaron los datos de consumos en kilogramos $(\mathrm{kg})$ de cada artesano para cada una de las materias primas en el año 2019. Se agruparon las demandas por mes y materias primas, para lograr una disminución de la variabilidad presentada con el consumo en periodos más cortos. Los resultados de la agrupación se encuentran en el Cuadro 1.

Cuadro 1

Consumos por materia prima en $\mathrm{kg} / \mathrm{mes}$

\begin{tabular}{|c|c|c|c|}
\hline Mes & Lana Natural & Lana Sintética & Media Lana \\
\hline 1 & 150 & 79 & 27 \\
\hline 2 & 169 & 88 & 35 \\
\hline 3 & 166 & 96 & 40 \\
\hline 4 & 143 & 62 & 28 \\
\hline 5 & 153 & 76 & 39 \\
\hline 6 & 167 & 56 & 24 \\
\hline 7 & 150 & 79 & 27 \\
\hline 8 & 169 & 88 & 35 \\
\hline 9 & 166 & 96 & 40 \\
\hline 10 & 143 & 62 & 28 \\
\hline 11 & 153 & 76 & 39 \\
\hline 12 & 167 & 56 & 24 \\
\hline
\end{tabular}

Fuente: elaboración propia

Se analizaron los datos de consumo de cada materia prima mediante el uso de estadística descriptiva en datos agrupados y sin agrupar, obteniendo medidas como la media, desviación estándar, coeficiente de variación, entre otras. Además, se realizaron pruebas de bondad y ajuste utilizando el software STAT FIT para determinar el comportamiento de consumo de cada materia prima, con un nivel de significancia del 95\%. En el Cuadro 2 se relacionan las distribuciones de probabilidad que se ajustan al comportamiento de cada materia prima. 
Cuadro 2

Distribuciones de probabilidad

materias primas seleccionadas

\begin{tabular}{|c|c|c|c|}
\hline \multirow{2}{*}{ Materia Prima } & \multirow{2}{*}{ Distribución de Probabilidad } & \multicolumn{2}{|c|}{ Parámetros } \\
\cline { 3 - 4 } & & Media (kg) & Desviación (kg) \\
\hline Lana Natural & Normal & 158,00 & 9,93 \\
\hline Lana Sintética & Normal & 76,10 & 14,00 \\
\hline Media Lana & Normal & 31,80 & 6,01 \\
\hline
\end{tabular}

Fuente: elaboración propia

\subsection{Estimación de costos}

En esta sección se estiman los costos que se tendrán en cuenta en el sistema de gestión de abastecimiento de APROARTES: costo de compra (C1), costo de ordenar (C2) y costo de mantenimiento (C3); teniendo en cuenta que una de las políticas de la asociación es no permitir faltantes.

Para estimar C1 fue necesario realizar contacto y cotizaciones con los diferentes proveedores de las materias primas seleccionadas. Sin embargo, debido a los lineamientos de APROARTES, la lana natural se comprará a los artesanos de la provincia del Sugamuxi y se definirá un precio estándar el cual será el C1 de esta materia prima. Para el resto de materias primas se tiene que los costos por proveedor mayorista son los que se encuentran en el Cuadro 3, donde se contemplan 5 proveedores para lana sintética y tres para la media lana; cabe resaltar que no se tienen más proveedores, debido a que varios proveedores cerraron sus empresas o no están en funcionamiento debido a la pandemia causada por el Covid 19.

Cuadro 3

Costo de compra materias primas (miles\$ $/ \mathrm{kg}$ )

\begin{tabular}{|c|c|c|c|c|c|}
\hline Materia Prima & P1 & P2 & P3 & P4 & P5 \\
\hline Lana Sintética & 58 & 45 & 49.5 & 61 & 15 \\
\hline Media Lana & 115 & 100 & 110 & - & - \\
\hline
\end{tabular}

Fuente: elaboración propia

Para estimar el C2, fue necesario definir las diferentes actividades que son necesarias para realizar un pedido. Por lo tanto, se definieron componentes del C2 y los tiempos necesarios para desarrollar cada componente y se calculó el costo de una hora de trabajo de un trabajador, teniendo como salario base el salario mínimo más prestaciones en el país de Colombia, obteniendo como los costos por componente mostrados en el Cuadro 4. Se decide sumar $\$ 10.000$ por concepto de papelería, por lo que, se obtiene un C2 por valor de $\$ 102.480$.

Cuadro 4

Tiempo y costo

por componentes

\begin{tabular}{|c|c|c|}
\hline Componente & Tiempo (h) & Costo (miles \$) \\
\hline Definir cantidades a pedir & 1,0 & 7,7 \\
\hline Definir colores & 2,5 & 19,2 \\
\hline Realización de pedido & 0,5 & 3,8 \\
\hline Recepción de pedido y entrega & 8,0 & 61,6 \\
\hline Papelería e insumos & 0,0 & 10,0 \\
\hline Total & 12,0 & 102,5 \\
\hline
\end{tabular}

Fuente: elaboración propia 
Por último, se realizó la estimación del C3 para cada una de las materias primas seleccionadas, debido a que los costos de las materias primas son diferentes. Por lo tanto, para calcular el C3 se tuvieron en cuenta los siguientes aspectos:

- Alquiler almacenes con sus servicios públicos: para la lana natural, se estimó el costo mensual de alquiler con sus servicios públicos para tres almacenes, donde la cantidad de almacenes surge como resultado del modelo que se analizará en la sección selección de proveedores. Se cargará al costo de cada kg de lana mensualmente, el costo de alquiler de almacenes con sus servicios públicos dividido por el inventario promedio de los almacenes; para la lana sintética y media lana, solo se considera un almacén, por lo que, se cargará al costo de cada kg de lana sintética o media lana, el costo de alquiler de almacén con sus servicios públicos dividido por el inventario promedio del almacén (este inventario se calculó como la capacidad media de los almacenes, dado que APROARTES no cuenta con datos de sus inventarios).

- Mano de obra manejo de inventarios: para la lana natural, se estimó a partir del tiempo requerido de mano de obra para realizar las actividades para el manejo de inventarios en los almacenes, y para la lana sintética y media lana, debido a que comparten el mismo almacén, se estimó el tiempo requerido de mano de obra para realizar las actividades para el manejo de inventarios en todo el almacén; este costo se dividirá en el inventario promedio total del almacén (este inventario se calculó como la capacidad media de los almacenes, dado que APROARTES no cuenta con datos de sus inventarios).

- Costo de capital: este costo se estimó multiplicando el costo de un kg por la tasa esperada de rentabilidad, para cada una de las materias primas seleccionadas.

Como resultado se obtuvo el C3 para las materias primas seleccionadas, los cuales se muestran en el Cuadro 5.

Cuadro 5

Costo de mantenimiento

(miles\$ /kg-mes)

\begin{tabular}{|c|c|}
\hline Materia Prima & C3 \\
\hline Lana natural & 1,353 \\
\hline Lana Sintética & 1,558 \\
\hline Media Lana & 2,658 \\
\hline
\end{tabular}

Fuente: elaboración propia

\subsection{Selección de proveedores}

Para realizar la selección de los proveedores para las materias primas seleccionadas se utilizó el método Pugh. Sin embargo, para la selección de los proveedores de la materia prima lana natural se tuvieron en cuenta las políticas propuestas por la junta directiva de APROARTES. Por lo tanto, la selección de proveedores para la materia prima lana natural se realizará únicamente por la ubicación de los artesanos, dado que los precios de compran se definirán entre APROARTES y los proveedores que cumplan con los tiempos de entrega y calidad requeridos. Además, por la característica de la materia prima lana natural, no es posible que un solo proveedor este en la capacidad de suplir todos los requerimientos.

Por lo mencionado anteriormente, se procede a identificar las ubicaciones donde se realizará la recepción y compra de la materia prima lana natural. Primero se identificó la ubicación de los 19 artesanos que hacen parte del sistema de abastecimiento, obteniendo la presencia en 7 municipios de la provincia del Sugamuxi. En la 
Figura 3 se encuentran los porcentajes de consumo de lana natural por municipio, donde se identifica claramente que Sogamoso $(43,9 \%)$ e Iza $(26,4 \%)$ son los municipios con mayor consumo de lana natural.

Figura 3

Porcentaje de consumo

de lana por municipio

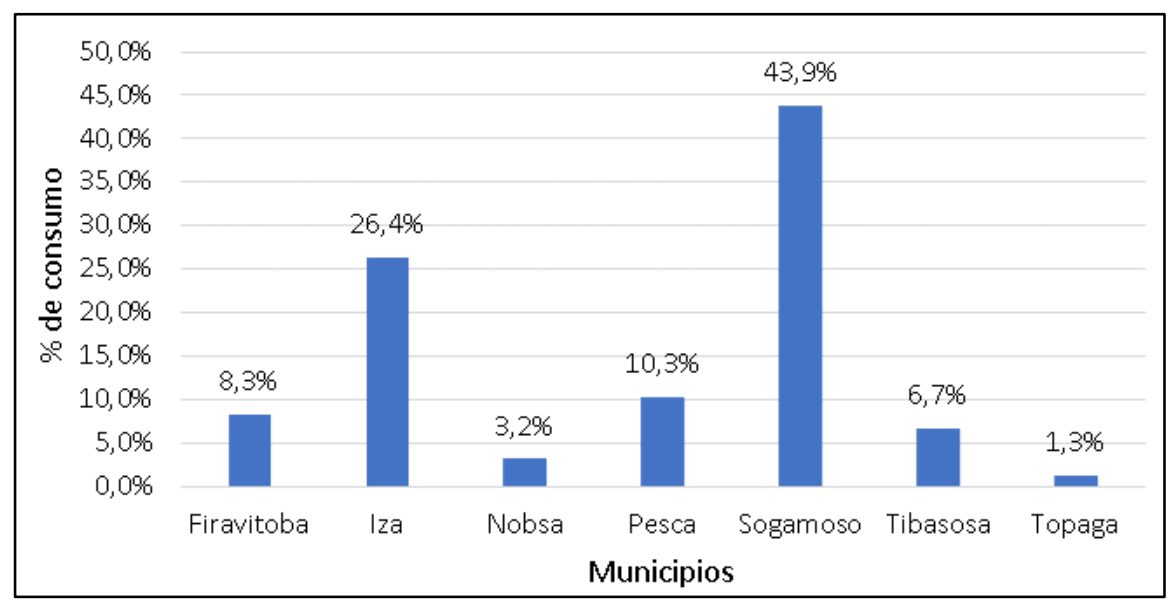

Fuente: elaboración propia

Se procede a utilizar un modelo de programación lineal entera mixta para identificar los municipios que servirán como puntos de recepción y almacenamiento de lana natural, y se identificaran los municipios a atender por cada punto de recepción y almacenamiento. El modelo de programación lineal mixta tiene como objetivo disminuir los costos fijos de almacenamiento y los costos variables de transporte. El modelo de programación lineal entera mixta es el siguiente:

- Índices: I para los nodos de origen y J para los nodos de destino.

- Variables: X (binaria), define si se utiliza a un origen para atender un destino;

$Y$ (binaria), define si se utiliza el punto de recepción y almacenamiento;

$\mathrm{CT}$, costo total.

- Parámetros: CKM, costo para transportar un $\mathrm{km} / \mathrm{kg}$ de lana;

$D$, distancia entre los nodos en $\mathrm{km}$;

CAL, costo fijo por punto de recepción y almacenamiento (\$);

OFER, cantidad (Kg) de lana que ofrece cada punto de recepción y almacenamiento;

DEM, cantidad de lana que requiere cada municipio en $\mathrm{kg}$;

NAL, número máximo de puntos de recepción y almacenamiento a abrir.

- Función objetivo: minimizar los costos fijos de almacenamiento y los costos variables de transporte.

$$
\operatorname{Min} C T=\sum_{I J} C K M * D E M_{J} * X_{I J}+C A L * Y_{I}
$$

- Restricciones: la ecuación 2 contiene restricciones de oferta, la ecuación 3 restricciones de demanda, e cuación 4 restricción de número máximo de puntos de recepción y almacenamiento.

$$
\begin{aligned}
& \sum_{J} D E M_{J} * X_{I J} \leq \text { OFER }_{I} * Y_{I} \quad ¥ I \\
& \sum_{I} D E M_{J} * X_{I J} \geq D E M_{J} \quad ¥ J
\end{aligned}
$$




$$
\sum_{I} Y_{I} \leq N A L
$$

El modelo de programación lineal entera mixta se resolvió utilizando el software Lingo, obteniendo como resultado que se deben de abrir puntos de recepción y almacenamiento en los municipios de Iza, Pesca y Sogamoso y se identificaron los municipios que debe atender cada punto de recepción y almacenamiento, siendo: Iza, a Iza; Pesca, a Firavitoba y Pesca; Sogamoso, a Nobsa, Sogamoso, Tibasosa y Tópaga.

Para seleccionar los proveedores para lana sintética y media lana, se utilizó el método Pugh, para lo cual se seleccionaron los siguientes criterios: precio, calidad, tiempos de entrega, disponibilidad de materias primas y pedidos mínimos y se asignó un valor de ponderación a cada criterio (García Gómez, 2013). En el Cuadro 6 se encuentran las asignaciones de valor y pesos de ponderación a asignar a los criterios de evaluación; se asignaron los siguientes pesos: precio (3), calidad (3), tiempos de entrega (2), disponibilidad de materias primas (3) y pedidos mínimos (1).

\section{Cuadro 6}

Valores de ponderación criterios

\begin{tabular}{|c|c|}
\hline Asignación de valor & Peso \\
\hline No es importante & 0 \\
\hline Poco importante & 1 \\
\hline Importante & 2 \\
\hline Muy importante & 3 \\
\hline
\end{tabular}

Fuente: elaboración propia

En el Cuadro 7 se encuentra la evaluación del método Pugh para la lana sintética, colocando + si el proveedor cumple por encima del criterio fijado, - si el proveedor no cumple con el criterio fijado o 0 si lo cumple. Complementariamente, en el Cuadro 8 se encuentran los resultados de la ponderación de los criterios, de donde se puede observar que el proveedor con el mejor ranking es P2, y a su vez, es el proveedor seleccionado para la compra de lana sintética.

Cuadro 7

Matriz de evaluación lana sintética

\begin{tabular}{|c|c|c|c|c|c|}
\hline Criterios & P1 & P2 & P3 & P4 & P5 \\
\hline Precio & 0 & + & + & 0 & + \\
\hline Calidad & + & + & + & + & + \\
\hline Tiempos de entrega & + & + & + & + & + \\
\hline Disponibilidad de MP & + & + & + & + & + \\
\hline Pedidos mínimos & + & + & - & + & - \\
\hline Suma Negativos & 0 & 0 & 1 & 0 & 1 \\
\hline Suma Positivos & 4 & 5 & 4 & 4 & 4 \\
\hline Suma General & 4 & 5 & 5 & 4 & 5 \\
\hline
\end{tabular}

Fuente: elaboración propia
Cuadro 8

Matriz de ponderación lana sintética

\begin{tabular}{|c|c|c|c|c|c|c|}
\hline Criterios & Pon & P1 & P2 & P3 & P4 & P5 \\
\hline Precio & 3 & 0 & 3 & 3 & 0 & 3 \\
\hline Calidad & 3 & 3 & 3 & 3 & 3 & 3 \\
\hline Tiempos de entrega & 2 & 2 & 2 & 2 & 2 & 2 \\
\hline Disponibilidad de MP & 3 & 3 & 3 & 3 & 3 & 3 \\
\hline Pedidos mínimos & 2 & 2 & 2 & -2 & 2 & -2 \\
\hline \multicolumn{2}{|c|}{ Suma Ponderada } & 10 & 13 & 9 & 10 & 9 \\
\hline \multicolumn{2}{|c|}{ Ranking } & 2 & 1 & 3 & 2 & 3 \\
\hline
\end{tabular}

Fuente: elaboración propia

En el Cuadro 9 se encuentra la matriz de evaluación del método Pugh para la media lana, y en el Cuadro 10 se encuentran los resultados de la ponderación de los criterios, de donde se puede observar que el proveedor con el mejor ranking es P2, y a su vez, es el proveedor seleccionado para la compra de la media lana. 
Cuadro 9

Matriz de evaluación media lana

\begin{tabular}{|c|c|c|c|c|}
\hline Criterios & P1 & P2 & P3 & P4 \\
\hline Precio & 0 & + & + & - \\
\hline Calidad & + & + & + & + \\
\hline Tiempos de entrega & + & + & + & 0 \\
\hline Disponibilidad de MP & + & + & + & 0 \\
\hline Pedidos mínimos & + & + & - & - \\
\hline Suma Negativos & 0 & 0 & 1 & 2 \\
\hline Suma Positivos & 4 & 5 & 4 & 1 \\
\hline Suma General & 4 & 5 & 5 & 3 \\
\hline
\end{tabular}

Fuente: elaboración propia
Cuadro 10

Matriz de ponderación media lana

\begin{tabular}{|c|c|c|c|c|c|}
\hline Criterios & Pon & P1 & P2 & P3 & P4 \\
\hline Precio & 3 & 0 & 3 & 3 & -3 \\
\hline Calidad & 3 & 3 & 3 & 3 & 3 \\
\hline Tiempos de entrega & 2 & 2 & 2 & 2 & 0 \\
\hline Disponibilidad de MP & 3 & 3 & 3 & 3 & 0 \\
\hline Pedidos mínimos & 2 & 2 & 2 & -2 & -2 \\
\hline Suma Ponderada & 10 & 13 & 9 & -2 \\
\hline Ranking & 2 & 1 & 3 & 4 \\
\hline
\end{tabular}

Fuente: elaboración propia

\subsection{Políticas de gestión de inventarios}

La política de gestión de inventarios teniendo como concepto base el VMI, se propondrá utilizando modelos determinísticos o modelos estocásticos, donde la decisión de utilizar un modelo, se tomará según el valor del coeficiente de variación de la demanda para cada materia prima; si el coeficiente de variación es menor a 0,2 se utilizará un modelo determinístico y si es mayor a 0,2 un modelo estocástico (Vidal Holguín, 2010).

Se calculó el coeficiente de variación de la demanda para cada materia prima, obteniendo los siguientes resultados: lana natural $(0,065)$, lana sintética $(0,191)$ y medía lana $(0,1977)$. De lo anterior, se puede concluir que para las tres materias primas se podría utilizar un modelo determinístico para determinar la política de gestión de inventarios, dado que el coeficiente de variación es menor a 0,2. Sin embargo, dado que el coeficiente de variación de la lana sintética y media lana está muy cerca al límite de decisión, también se utilizará un modelo estocástico para determinar la política de gestión de inventarios de estás dos materias primas.

Con relación a los modelos determinísticos, se formularon dos modelos:

i. Un modelo matemático de programación entera mixta multi periodo, multi producto y multi proveedores, para definir las cantidades a pedir para cada uno de los meses a tener en consideración, para definir las cantidades en inventario y proveedores a los cuales comprar las materias primas. El modelo, fundamentado en un modelo programación de producción, es el siguiente:

- Índices: I para la materia primas, T para el periodo y P para el proveedor.

- Variables: $\mathrm{Q}$, cantidades ( $\mathrm{kg}$ ) a pedir de cada materia prima por periodo y por proveedor; INV, cantidades $(\mathrm{kg})$ a mantener en inventario de cada materia prima por periodo; $\mathrm{N}$ (binaria), define si se pide la materia prima en un periodo y aun proveedor; CT costo total del ejercicio.

- Parámetros: $\mathrm{C} 1$, precio $(\$ / \mathrm{kg})$ de compra de la materia prima a los proveedores;

$\mathrm{C} 2$, costo de ordenar (\$/orden);

C3, costo de mantener en inventario $(\$ / \mathrm{kg}-\mathrm{mes})$ cada materia prima; PMIN, cantidad mínima $(\mathrm{kg})$ a pedir por materia prima y proveedor;

PMAX, cantidad máxima $(\mathrm{kg})$ a pedir por materia prima y proveedor; $\mathrm{D}$, demanda ( $\mathrm{kg} / \mathrm{mes}$ ) de las materias primas en cada periodo; CPMAX, capacidad $(\mathrm{kg})$ de inventario máximo.

- Función objetivo: minimizar costos de compra, ordenes de pedido y almacenamiento. 


$$
\operatorname{Min} C T=\sum_{I T P} C 1_{I P} * Q_{I T P}+C 2 * N_{I T P}+C 3_{I} * I N V_{I P}
$$

- Restricciones: la ecuación 6 contiene las restricciones de cantidad mínima a pedir, la ecuación 7 las restricciones de cantidad máxima a pedir, la ecuación 8 las restricciones de flujo, la ecuación 9 las restricciones de inventario máximo, la ecuación 10 las restricciones de inventario inicial y la ecuación 11 las restricciones de no negatividad.

$$
\begin{aligned}
& Q_{I T P} \geq P M I N_{I P} * N_{I T P} \quad ¥ I, P, T>0 \\
& Q_{I T P} \leq P M A X_{I P} * N_{I T P} \quad ¥ I, P, T>0 \\
& \sum_{P} Q_{I T P}+I N V_{I T-1}=D_{I T}+I N V_{I T} \quad ¥ I, T>0 \\
& \sum_{I} I N V_{I T} \leq C P M A X \quad ¥ T>0 \\
& I N V_{I T}=0 \quad ¥ I, T>0 \\
& Q_{I T P}, I N V_{I T} \geq 0 \quad ¥ I, P, T
\end{aligned}
$$

El modelo se resolvió utilizando el software Lingo, obteniendo los resultados presentados en el Cuadro 11 , donde se evidencian las cantidades a pedir al proveedor dos. Complementariamente, se obtuvo un valor del costo total anual de $\$ 177.571 .000$, sin embargo, también se calculó el costo total sin tener en cuenta el costo de compra, el cual es $\$ 3.686 .688$.

\section{Cuadro 11}

Resultados modelo programación lineal entera mixta $(\mathrm{kg})$

\begin{tabular}{|c|c|c|c|}
\hline Mes & Lana Natural & Lana Sintética & Media Lana \\
\hline Enero & 150 & 79 & 27 \\
\hline Febrero & 169 & 88 & 35 \\
\hline Marzo & 166 & 96 & 40 \\
\hline Abril & 143 & 62 & 28 \\
\hline Mayo & 153 & 76 & 39 \\
\hline Junio & 167 & 56 & 24 \\
\hline Julio & 150 & 79 & 27 \\
\hline Agosto & 169 & 88 & 35 \\
\hline Septiembre & 166 & 96 & 40 \\
\hline Octubre & 143 & 62 & 28 \\
\hline Noviembre & 153 & 76 & 39 \\
\hline Diciembre & 167 & 56 & 24 \\
\hline
\end{tabular}

Fuente: elaboración propia

ii. Modelo de programación lineal entera mixta tomando como base el modelo de transporte: este modelo fundamentado en un modelo de transporte, permitirá definir las cantidades a pedir y en inventario de cada una de las materias primas seleccionadas. El modelo es el siguiente: 
- Índices: I para los orígenes, J para los destinos y P el tipo de materia prima.

- Variables: $X$, cantidades $(\mathrm{kg})$ a pedir de cada materia prima por periodo;

$\mathrm{Y}$ (binaria), si se pide la materia prima en un periodo;

$\mathrm{CT}$, costo total del ejercicio.

- Parámetros: $\mathrm{C} 1$, precio de compra $(\$ / \mathrm{kg})$ de la materia prima al proveedor seleccionado;

$\mathrm{C} 2$, costo de ordenar (\$/orden);

C3, costo de mantener en inventario ( $\$ / \mathrm{kg}$-mes) de cada materia prima;

PMAX, cantidad máxima a pedir por materia prima $(\mathrm{kg})$;

$\mathrm{D}$, demanda de las materias primas en cada periodo en $(\mathrm{kg} / \mathrm{mes})$.

- Función objetivo: minimizar costos de compra, ordenes de pedido y almacenamiento, donde la sumatoria para calcular el costo de mantener solo se aplicará sí J>I.

$$
\operatorname{Min} C T=\sum_{I J P} C 1_{P} * X_{I J P}+C 2 * Y_{I P}+\sum_{I J P}(J-I) * C 3_{P} * X_{I J P}
$$

- Restricciones: la ecuación 13 restricciones de cantidad máxima a pedir según la demanda, la ecuación 14 restricciones de demanda, la ecuación 15 restricciones de cantidad máxima a pedir por el proveedor y la ecuación 16 restricciones de no negatividad.

$$
\begin{gathered}
\sum_{J \geq 1} X_{I J P} \leq\left(\sum_{J \geq I} D_{P, J}\right) * Y_{I, P} \quad ¥ I, P \\
\sum_{I \leq J} X_{I J P} \leq D_{P, J} \quad ¥ J, P \\
\sum_{J} X_{I J P} \leq P M A X_{P} \quad ¥ I, P \\
X_{I J P} \geq 0 \quad ¥ I, J, P
\end{gathered}
$$

El modelo se resolvió utilizando el software Lingo, obteniendo los resultados presentados en el Cuadro 12 , donde se evidencian las cantidades a pedir al proveedor dos. Se obtuvo un valor del costo total anual de $\$ 177.374 .600$, sin embargo, también se calculó el costo total sin tener en cuenta el costo de compra, el cual es $\$ 3.489 .635$.

Cuadro 12

Resultados modelo con base algoritmo de transporte(kg)

\begin{tabular}{|c|c|c|c|}
\hline Mes & Lana Natural & Lana Sintética & Media Lana \\
\hline Enero & 150 & 79 & 62 \\
\hline Febrero & 169 & 88 & - \\
\hline Marzo & 166 & 158 & - \\
\hline Abril & 143 & - & 63 \\
\hline Mayo & 153 & 132 & - \\
\hline Junio & 167 & - & 62 \\
\hline Julio & 150 & 79 & - \\
\hline Agosto & 169 & 88 & \\
\hline
\end{tabular}




\begin{tabular}{|c|c|c|c|}
\hline Mes & Lana Natural & Lana Sintética & Media Lana \\
\hline Septiembre & 166 & 158 & 68 \\
\hline Octubre & 143 & - & - \\
\hline Noviembre & 153 & 132 & 63 \\
\hline Diciembre & 167 & - & - \\
\hline
\end{tabular}

Fuente: elaboración propia

Con respecto a los modelos estocásticos, se utilizaron dos sistemas de gestión:

i. Sistema de revisión periódica: la cantidad económica de pedido mediante la ecuación 17, el tiempo entre pedidos la ecuación 18, la desviación de la demanda durante el tiempo de amortización la ecuación 19, las existencias de seguridad la ecuación 20, el nivel del inventario inicial la ecuación 21, el nivel del inventario promedio en la ecuación 22 y el costo total anual la ecuación 23.

$$
\begin{gathered}
Q=\sqrt{\frac{2 * C 2 * D_{P}}{C 3}} \\
t=\frac{Q}{D_{P}} \\
\sigma_{1}=\sigma * \sqrt{L T+t} \\
E S=Z_{\propto} * \sigma_{1} \\
N I I=Q+E S \\
N I P=\frac{Q}{2}+E S \\
C T=\left(C 1 * D_{P}+C 2 * \frac{D_{P}}{Q}+C 3 * \frac{Q}{2}\right) * 12
\end{gathered}
$$

Donde: $D_{P}$, es la demanda promedio de la materia prima; $\sigma$, es la desviación estándar de la demanda; LT es el tiempo de entrega de los pedidos ( 5 días) y $Z_{\propto}$ es el valor obtenido de una distribución normal estándar inversa con un nivel de significacia de $\propto$.

El modelo se resolvió utilizando el software Excel, obteniendo los resultados presentados en el Cuadro 13, donde se evidencian las cantidades económicas de pedido $Q$ a pedir al proveedor dos, los tiempos entre pedidos $t$, las existencias de seguridad ES, los niveles de inventario promedio NIP y los niveles de inventario incial NII. Complementariamente, se obtuvo un valor del costo total anual de $\$ 181.518 .449$, sin embargo, también se calculó el costo total sin tener en cuenta el costo de compra, el cual es $\$ 7.464 .449$.

Cuadro 13

Resultados Sistema de revisión periódica

\begin{tabular}{|c|c|c|c|}
\hline Variable & Lana Natural & Lana Sintética & Media Lana \\
\hline $\mathrm{Q}(\mathrm{kg})$ & 155 & 101 & 50 \\
\hline $\mathrm{t}$ (meses) & 0,98 & 1,31 & 1,57 \\
\hline $\mathrm{ES}(\mathrm{kg})$ & 21 & 34 & 16 \\
\hline NIP (kg) & 99 & 85 & 41 \\
\hline NII (kg) & 176 & 135 & 66 \\
\hline
\end{tabular}

Fuente: elaboración propia 
ii. Modelo pedido conjunto: en este modelo se determina un tiempo entre pedidos igual para todas las materias primas. Donde se calcula el tiempo entre pedidos mediante la ecuación 24 , la desviación estándar con la ecuación 25 , las existencias de seguridad con la ecuación 26 , la cantidad económica de pedido con la ecuación 27 , el nivel promedio de inventario con la ecuación 28 , la cantidad a pedir con la ecuación 29 y el costo total con la ecuación 30.

$$
\begin{gathered}
T=\sqrt{\frac{2 *\left(O+\sum_{i} C 2_{i}\right)}{I \sum_{i} C 1_{i} * D_{i}}} \\
s^{\prime}{ }_{d}=s_{d} * \sqrt{L T+T} \\
E S_{i}=Z_{i} * s^{\prime}{ }_{d} \\
Q_{i}=D_{i} * T \\
N I P=\frac{Q_{i}}{2}+E S_{i} \\
C P=Q_{i} \\
C T=\left(\sum_{i} C 1_{i} * D_{i}+\frac{C 2}{T}+\sum_{i} C 3_{i} *\left(\frac{Q_{i}}{2}+E S_{i}\right)\right) * 12
\end{gathered}
$$

Donde: $D_{i}$, es la demanda promedio de la materia prima i; $s_{d}$, es la desviación estándar de la demanda de cada materia prima; LT es el tiempo de entrega de los pedidos ( 5 días) y $Z_{\alpha}$ es el valor obtenido de una distribución normal estándar inversa con un nivel de significacia de $\propto$.

El modelo se resolvió utilizando el software Excel, obteniendo como resultado pedir cada 25 días las tres materias primas, y su vez, se obtuvieron los resultados en el Cuadro 14, donde se evidencian la desviación de las materias primas $s^{\prime} d$, las cantidades económicas de pedido $Q$, las existencias de seguridad ES, los niveles de inventario promedio NIP y las cantidades a pedir CP.Además, se obtuvo un valor del costo total anual de \$178.265.674, sin embargo, también se calculó el costo total sin tener en cuenta el costo de compra, el cual es $\$ 4.211 .674$.

Cuadro 14

Resultados modelo pedido conjunto (kg)

\begin{tabular}{|c|c|c|c|}
\hline Variable & Lana Natural & Lana Sintética & Media Lana \\
\hline s'd & 11 & 15 & 7 \\
\hline ES & 22 & 30 & 14 \\
\hline Q & 135 & 65 & 27 \\
\hline NIP & 89 & 62 & 28 \\
\hline CP & 135 & 65 & 27 \\
\hline
\end{tabular}

Fuente: elaboración propia

Para seleccionar la mejor política para realizar la gestión de inventarios en APROARTES, se consolidan en el Cuadro 15 los resultados de costo de cada modelo. 
Cuadro 15

Consolidación de resultados (millones\$)

\begin{tabular}{|c|c|c|}
\hline Modelo & Costo Total & Costo Total sin C1 \\
\hline Programación Entera Mixta & 177,571 & 3,687 \\
\hline Base problema del transporte & 177,375 & 3,490 \\
\hline EOQ Periódico & 181,518 & 7,464 \\
\hline Conjunto & 178,265 & 4,211 \\
\hline
\end{tabular}

Fuente: elaboración propia

Dado lo anterior, la política que minimiza los costos por gestión de inventarios para las materias primas seleccionadas, es la política generada a partir del modelo de programación lineal que tiene como base el modelo de transporte, por lo que, los costos asociados a ordenar y mantener inventario son menores, y a su vez, estos hacen que el costo total sea menor. Por lo que, la política de gestión de inventarios para: lana natural, pedir la cantidad requerida de la demanda del mes cada mes como se evidencia en el Cuadro 12; lana sintética y media lana, en algunos meses se pedirá solamente la demanda del mes, en otros meses se pedirá la demanda de varios meses y en otros meses no se pedirá, las cantidades a pedir en cada uno de los meses se encuentran en el Cuadro 12 ; teniendo un costo anual de $\$ 177.374 .600$.

\section{Conclusiones}

Se identificó que APROARTES produce 175 productos representativos, para los cuales utilizan aproximadamente 92 materias primas o insumos. Sin embargo, dentro de estas 92 materias primas o insumos que se utilizan, se tienen 10 materias primas o insumos comunes, las cuales son: lana natural, lana sintética, media lana, productos de la naturaleza, madera, tela, hilo, algodón, fique y semillas. Se seleccionaron las materias primas que formaran parte del sistema de gestión de abastecimiento: lana natural, lana sintética y media lana y se determina el comportamiento de las materias primas para analizar de forma más exacta los modelos utilizados para el diseño del sistema de gestión de abastecimiento.

El modelo que disminuyó los costos del sistema de gestión de inventarios de APROARTES es el modelo determinístico que toma como base el problema del transporte, siendo la política generada por este modelo la seleccionada. La política de gestión de inventarios recomendada para el sistema de gestión de abastecimiento colaborativo de APROARTES será la siguiente: para la lana natural, pedir la cantidad requerida de la demanda del mes cada mes como se evidencia en el Cuadro 12; para el caso de la lana sintética y media lana, en algunos meses se pedirá solamente la demanda del mes, en otros meses se pedirá la demanda de varios meses y en los meses restantes no se pedirá nada, las cantidades a pedir en cada uno de los meses se encuentran en el Cuadro 12; teniendo un costo anual de $\$ 177.374 .600$.

\section{Referencias bibliográficas}

Aguilar Santamaría, P. A. (2012). Un modelo de clasificación de inventarios para incrementar el nivel de servicio al cliente y la rentabilidad de la empresa. Pensamiento \& Gestión, (32), 142-164.

Alfonso, L. (2018). Procedimiento de Evaluación y Selección de Proveedores Basado en el Proceso de Análisis Jerárquico y en un Modelo de Programación Lineal Entera Mixta. Ingeniería, 23(3), 230-251. https://doi.org/10.14483/23448393.13316

Amaro Castillo, A. G. (2017). Análisis estratégico para la selección de proveedores de servicios de autotransporte de carga (Doctoral dissertation, Universidad Autónoma de Nuevo León). 
Arango-Serna, M., Adarme-Jaimes, W., \& Zapata-Cortes, J. (2010). Gestión cadena de abastecimiento - logística con indicadores bajo incertidumbre, caso aplicado sector panificador Palmira. Ciencia e Ingeniería Neogranadina, 20(1), 97-115.

Arias-Vargas, M. (2017). Impacto en el inventario de seguridad por la utilización de la desviación estándar de los errores de pronóstico. Revista Tecnología En Marcha, 30(1), 49. https://doi.org/10.18845/tm.v30i1.3064

Barcelli-Gómez, G. (2017). Gestión, planificación y control de la producción. Ingeniería Industrial. https://doi.org/10.26439/ing.ind1999.n024.523

Bustamante-Salazar, A. M. (2017). Costeo basado en actividades -ABC: revisión de literatura. Revista CEA. https://doi.org/10.22430/24223182.72

Castellano De La Cruz, N. A., Gamarra Vengoechea, A. M., \& Garcia Luenga, J. T. (2017). Simulación de un modelo costo-eficiente de reabastecimiento conjunto inventario (CJRP).

Díaz-Batista, J., \& Pérez-Armayo, D. (2012). Optimización de los niveles de inventario en una cadena de suministro. Rev. Ingeniería Industrial, 33(2), 126-132.

Duran, Y. (2012). Administración del inventario: elemento clave para la optimización de las utilidades en las empresas. Visión Gerencial.

Escobar, J. W., Linfati, R., \& Jaimes, W. A. (2017). Gestión de Inventarios para distribuidores de productos perecederos. Ingeniería y Desarrollo. https://doi.org/http://dx.doi.org/10.14482/inde.35.1.8950

García Gómez, A. (2013). Metodología para la evaluación y selección de proveedores de servicios de mantenimiento (Bachelor's thesis, Universidad Autónoma de Occidente).

Gómez, R. A., Cano, J. A., \& Campo, E. A. (2016). Selección de proveedores en la minería de oro con lógica difusa. Revista Venezolana de Gerencia, 21(75). https://doi.org/10.31876/revista.v21i75.21897

Gutiérrez, V., \& Rodríguez, L. (2008). Diagnóstico regional de gestión de inventarios en la industria de producción y distribución de bienes Regional diagnostic of inventory management in the productiondistribution industry. Rev. Fac. Ing. Univ. Antioquia N. ${ }^{\circ}$, 45, 15-26.

https://doi.org/10.1177/000348947908800223

Lancheros, D., Mojica, A., \& Barbosa, D. (2015). Sistema de información para administración de inventarios basado en técnicas de minería de datos. Information System for Inventory Management Based on Data Mining Techniques., (24), 143-173. Retrieved from http://search.ebscohost.com/login.aspx?direct=true \&db=a9h\&AN=118525321\&lang=es\&site=edslive $\&$ scope $=$ site $\&$ custid $=$ s4608367

Muñoz Claro, T. (2014). Fundamentos de las metodologías AHP y ANP. Aplicación al problema de selección de proveedores para la elaboración de una cerveza artesanal. Universidad de Sevilla. Recuperado de: http://zaguan.unizar.es/TAZ/EUCS/2014/14180/TAZ-TFG-2014-408.pdf

Osorio, C. A. (2015). Modelos para el control de inventarios en las pymes. Panorama. https://doi.org/10.15765/pnrm.v2i6.241

Palacio-León, O., Barrios-León, M., \& Adarme-Jaimes, W. (2018). Modelo de Coordinación de Inventarios Colaborativos Soportado en un Enfoque Sociotécnico de Sistemas. Ingeniería Investigación y Desarrollo. https://doi.org/10.19053/1900771x.v18.n1.2018.8204 
Parga-Prieto, A. T., \& Aranda-Pinilla, J. A. (2018). Política de inventario para demandas con tendencia y aleatoriedad. Caso comercializadora de lubricantes. Revista Inventum, 13(24), 50.

Peña, O., \& Silva, R. (2015). Factores incidentes sobre la gestión de sistemas de inventario en organizaciones venezolanas. Telos: Revista de Estudios Interdisciplinarios En Ciencias Sociales, 18(2), 187-207.

Pereira, D. F., Oliveira, J. F., \& Carravilla, M. A. (2020). Tactical sales and operations planning: A holistic framework and a literature review of decision-making models. International Journal of Production Economics, 228 (Octuber 2020). https://doi.org/10.1016/j.ijpe.2020.107695

Pérez-Vergara, I., Cifuentes-Laguna, A. M., Vásquez-García, C., \& Marcela-Ocampo, D. (2013). Un modelo de gestión de inventarios para una empresa de productos alimenticios. Ingeniería Industrial, 34(2), 227-236. Retrieved from http://scielo.sld.cu/scielo.php?script=sci_arttext\&pid=S1815$59362013000200011 \& \operatorname{lng}=e s \& n r m=i s o \&$ tIng=es

Razmi, J., Hosseini Rad, R., \& Sangari, M. S. (2010). Developing a two-echelon mathematical model for a vendor-managed inventory (VMI) system. International Journal of Advanced Manufacturing Technology, 48(5-8), 773-783. https://doi.org/10.1007/s00170-009-2301-7

Rodríguez, E. C. (2015). Modelo de inventarios para control económico de pedidos en empresa comercializadora de alimentos. Revista de Ingenierías: Universidad de Medellín, 14(27), 163-177.

Sablón-Cossío, N., Acevedo-Urquiaga, A. J., Acevedo-Suárez, J. A., \& Medina-León, A. (2015). Propuesta para la evaluación de la planificación colaborativa de la cadena de suministro. Ingeniería Industrial, 36(1), 580597.

Salas-Navarro, K., Maiguel-Mejía, H., \& Acevedo-Chedid, J. (2017). Metodología de Gestión de Inventarios para determinar los niveles de integración y colaboración en una cadena de suministro. Ingeniare. Revista Chilena de Ingeniería, 25(2), 326-337. https://doi.org/10.4067/s0718-33052017000200326

Sánchez, D., \& Ramírez, N. (2018). Diseño de un modelo para la administración de inventarios en un cultivo de fresa, basado en el modelo de pedido para un solo periodo y las métricas 6 sigma. Ingeniería y Competitividad, 105(1), 95-105.

Silador Utrera, E., Naranjo García, M. M., Marrero Marrero, M., Utrera Velázquez, A. I., \& Rodríguez Pacha, E. V. (2015). Propuesta de modelo matemático de gestión de inventario. Caso Servi Cupet Punta Gorda, Cienfuegos, Cuba Inventory management proposed mathematical model. Caso Servi Cupet Punta Gorda, Cienfuegos, Cuba. Ciencia, Tecnología e Innovación.

Southard, P. B., \& Swenseth, S. R. (2008). Evaluating vendor-managed inventory (VMI) in non-traditional environments using simulation. International Journal of Production Economics, 116(2), 275-287. https://doi.org/10.1016/j.ijpe.2008.09.007

Taboada-González, P., Aguilar-Virgen, Q., Ibarra-TrujiMo, J., \& Ramírez-Barreto, M. (2016). Optimización de un Sistema de Abastecimiento de Pintura a Concesionarios de Baja y Media Demanda. Información Tecnológica, 27(3), 53-60. https://doi.org/10.4067/S0718-07642016000300006

Tat, R., Taleizadeh, A. A., \& Esmaeili, M. (2015). Developing economic order quantity model for noninstantaneous deteriorating items in vendor-managed inventory (VMI) system. International Journal of Systems Science, 46(7), 1257-1268. 
Torrijos, M. G. (2018). La Selección de proveedores elementos clave en la Gestión de aprovisionamientos. Universidad Militar Nueva Granada, 63. Recuperado de: http://digibuo.uniovi.es/dspace/bitstream/10651/47803/3/TFM_MargaritaGilTorrijos.pdf

Vázquez Yánez, W. R. (2012). Modelo de gestión de inventarios para la empresa Martec Cía. Ltda.

Vidal Holguín, C. J. (2010). Fundamentos de control y gestión de inventarios. Fundamentos de control y gestión de inventarios. https://doi.org/10.25100/peu.48

Zanoni, S. Yaber, MY (2015). Una cadena de suministro de dos niveles con acuerdo de stock en consignación y demanda dependiente del stock. Revista internacional de investigación de producción, 53 (12), 3561-3572.

Esta obra está bajo una Licencia Creative Commons

Attribución-NoCommercial 4.0 International

\section{(cc) EY-NC}

